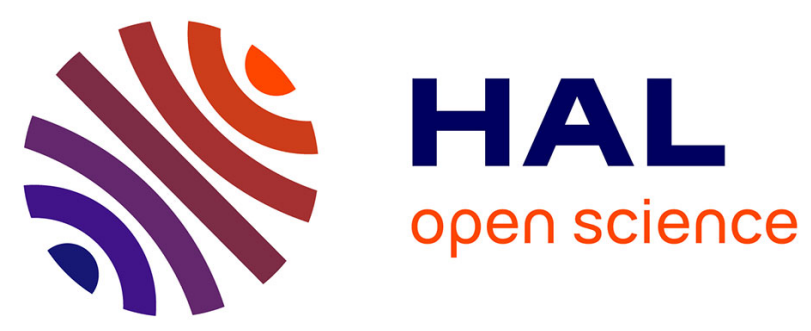

\title{
Un système à commande digitale pour l'étude des dispositifs semiconducteurs par le balayage d'un faisceau de lumière laser
}

Gh. A. Stanciu, I.M. Popescu, C.M. Stoichita

\section{- To cite this version:}

Gh. A. Stanciu, I.M. Popescu, C.M. Stoichita. Un système à commande digitale pour l'étude des dispositifs semiconducteurs par le balayage d'un faisceau de lumière laser. Revue de Physique Appliquée, 1980, 15 (1), pp.55-59. 10.1051/rphysap:0198000150105500 . jpa-00244698

\section{HAL Id: jpa-00244698 \\ https://hal.science/jpa-00244698}

Submitted on 1 Jan 1980

HAL is a multi-disciplinary open access archive for the deposit and dissemination of scientific research documents, whether they are published or not. The documents may come from teaching and research institutions in France or abroad, or from public or private research centers.
L'archive ouverte pluridisciplinaire HAL, est destinée au dépôt et à la diffusion de documents scientifiques de niveau recherche, publiés ou non, émanant des établissements d'enseignement et de recherche français ou étrangers, des laboratoires publics ou privés. 


\title{
Un système à commande digitale pour l'étude des dispositifs semiconducteurs par le balayage d'un faisceau de lumière laser
}

\author{
Gh. A. Stanciu, I. M. Popescu et C. M. Stoichita \\ Laboratoire de Physique, Institut Polytechnique de Bucarest, Roumanie
}

(Reçu le 30 août 1979, accepté le 10 octobre 1979)

\begin{abstract}
Résumé. - Il est présenté un système digital de balayage à laser pour l'investigation des dispositifs à semiconducteurs. Une discussion de l'équipement et de ses performances est donnée, ainsi que quelques applications qui mettent en évidence les possibilités de travail du système.
\end{abstract}

\begin{abstract}
A laser scanning digital system for investigations in semiconductor devices is shown. A discussion of the apparatus and the performance is given, and some applications which set up the work possibilities are presented.
\end{abstract}

1. Introduction. - L'utilisation du balayage automatique d'un faisceau de lumière émis par une source classique, pour l'étude des dispositifs semiconducteurs, a été traitée dans plusieurs travaux [1-3].

Divers systèmes de balayage ont ainsi été réalisés, qui permettent d'étudier les dispositifs à semiconducteurs [3-6], les matériaux semiconducteurs [7] ou la photoluminescence des diodes électroluminescentes [8].

En effet, les méthodes qui utilisent les faisceaux de lumière ont l'avantage d'être des méthodes non destructives et de permettre des études de dispositifs sous leurs conditions de fonctionnement. Dans ce domaine, l'utilisation d'un faisceau laser apporte les facilités suivantes :

- une meilleure focalisation due à la cohérence du faisceau,

- la possibilité de connaître avec précision la profondeur de la pénétration lumineuse (pour une concentration donnée de porteurs), par suite de son caractère monochromatique,

- la possibilité de travailler avec un niveau d'excitation convenable.

L'exploitation de la raie $\lambda=632,8 \mathrm{~nm}$ du laser à $\mathrm{He}-\mathrm{Ne}$ peut avoir, pour ces études, l'inconvénient d'une faible profondeur de pénétration dans le semiconducteur. Ceci ne permet donc pas dans certains cas d'explorer les régions désirées. Par contre, le développement actuel des dispositifs de haute fréquence, dont les jonctions se situent au voisinage de la surface, donne un intérêt à un tel faisceau de balayage.

Par ailleurs, comme l'ont signalé d'autres auteurs [2], [6], [9] les avantages des méthodes ci-dessus posent le problème de l'utilisation d'un calculateur lié au système de balayage.
Le but essentiel de notre travail a donc été de mettre au point et de réaliser, dans le cas d'un faisceau laser à $\mathrm{He}-\mathrm{Ne}$, un système à commande digitale, qui permet ce contrôle par calculateur.

2. Dispositif expérimental. -2.1 CIRCUIT ÉleCTRIQUE DU DISPOSITIF. - Le schéma électrique du dispositif réalisé est représenté dans la figure 1. Les deux déflecteurs (DV et $\mathrm{DH})$ ont des systèmes de commande identiques.

Le générateur de position (GP) envoie un signal digital sur les deux convertisseurs digitaux-analogiques (CDAH et CDAV). A la sortie des convertisseurs

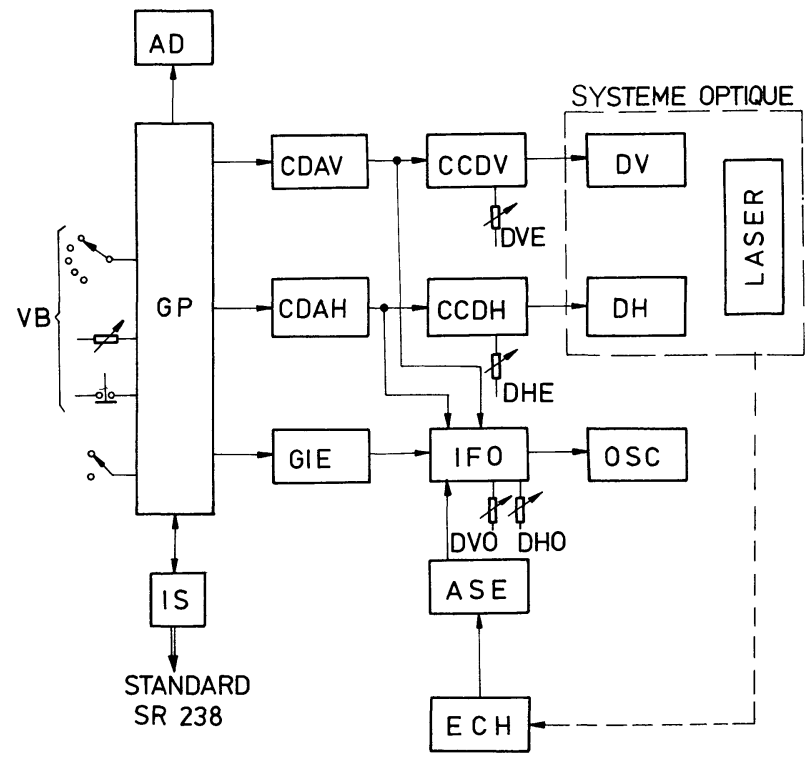

Fig. 1. - Schéma électrique du système de balayage par faisceau laser $(\lambda 632,8 \mathrm{~nm})$

[Electrical schematic of the laser scanning system.] 
les tensions obtenues correspondent aux coordonnées cartésiennes du spot sur la surface de l'échantillon $(\mathrm{ECH})$.

Les signaux obtenus à la sortie des convertisseurs digitaux-analogiques sont envoyés sur les circuits de commande des déflecteurs (CCDV et $\mathrm{CCDH}$ ). Les tensions de coordonnées sont transformées en courants de coordonnées pour l'attaque des petites résistances des boucles des déflecteurs. Le circuit de commande des déflecteurs assure aussi la correction des erreurs de position dans le cas d'une exploration rapide. De cette manière, sur l'échantillon est déroulée une trame qui peut être déplacée précisément à l'aide de deux potentiomètres (DVE et DHE).

Les mêmes signaux sont envoyés sur une interface de loscilloscope (IFO). Les tensions de coordonnées donnent sur l'écran de l'oscilloscope une trame qui est similaire à celle de l'échantillon. L'exploration se fait par le déroulement d'un cadre constitué de 100 lignes, chaque ligne ayant 100 points. Dans la figure 1, on voit que les signaux de sortie du générateur de position (GP) sont envoyés vers un bloc d'affichage décimal (AD). Ainsi, on peut assurer un enregistrement très précis, en coordonnées cartésiennes, de la position du spot sur l'échantillon.

La fréquence d'oscillateur du générateur de position est commandée de l'extérieur. Cette commande assure le réglage de la vitesse de balayage du spot (VB). Le générateur de position possède, pour travailler en association avec un calculateur, une interface standard (IS) SR 238. Les instructions spécifiques correspondantes ne font pas l'objet de cet article.

Un générateur d'extinction (GIE), situé dans le même bloc que GP, assure la disparition du spot sur l'écran pendant les courses inverses. Ce générateur est actionné par un commutateur en fixant le nombre des lignes affichées sur l'écran. On peut travailler en balayage automatique ( $100 \ldots 1$ lignes) à phase réglable, en exploration manuelle point par point ou en association avec le calculateur. En utilisant un petit nombre de lignes, on peut simplifier l'interprétation des résultats.

Le circuit de détection est aussi représenté sur la figure 1. Le signal donné par l'échantillon (photocourant ou phototension) est amplifié à l'aide d'un amplificateur vidéo, puis envoyé sur l'interface de l'oscilloscope. Ici, le signal peut être appliqué sur l'axe $O z$ de l'oscilloscope, et l'écran de celui-ci fournit ainsi une image photographique de la surface d'échantillon. De même, le signal peut être additionné à la tension de déflection verticale lors de l'observation des graphiques sur l'écran.

Nous avons utilisé un oscilloscope à mémoire OG 2-31 RFT, en remplaçant les tiroirs de celui-ci par le bloc de commande du système et par l'interface d'oscilloscope. La trame d'oscilloscope peut être déplacée à l'aide de potentiomètres DVO et DHO.

La vue générale du système que nous avons réalisé est présentée dans la figure 2.

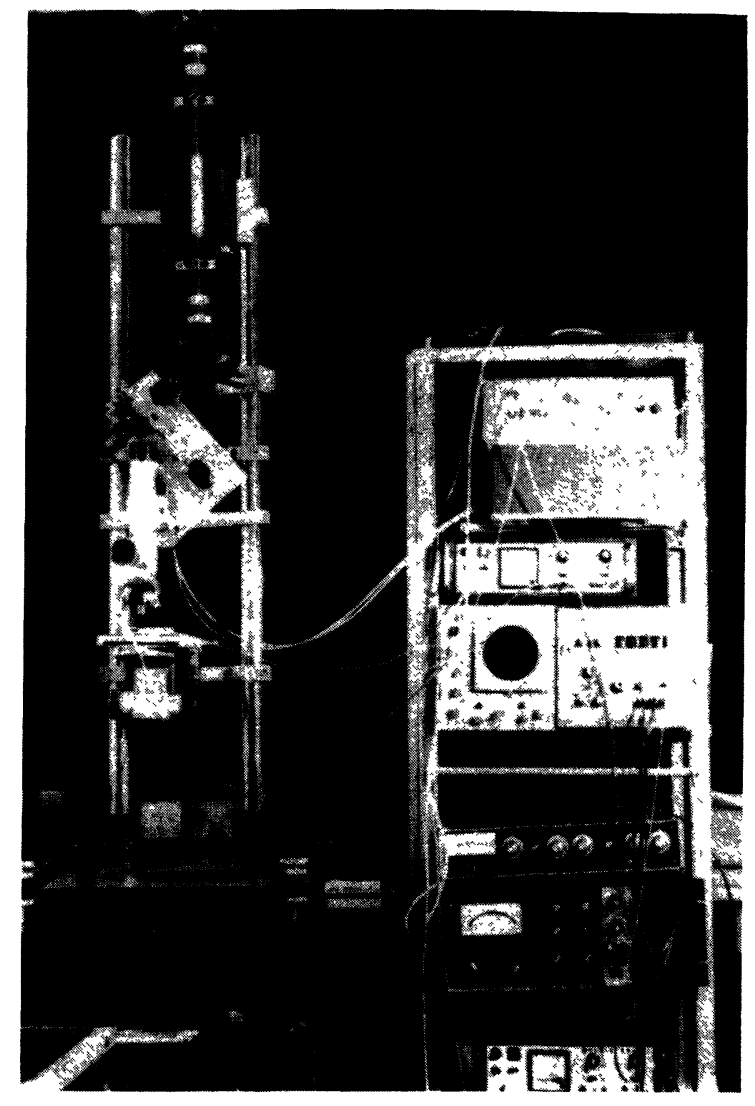

Fig. 2. - Vue générale du système réalisé

[General view of the realized system.]

2.2 Dispositif optiQue. - Le dispositif optique utilisé est décrit en détail dans la référence [9].

Nous avons utilisé un laser à He-Ne de type HNA50, Karl Zeiss Jena de puissance $5 \mathrm{~mW}$. L'association de deux galvanomètres permet une résolution de mieux que 300 points et une fréquence de résonance de $3000 \mathrm{~Hz}$. Des miroirs, constitués par des lames de microscope recouvertes d'un dépôt d'aluminium et de dimensions $0,8 \times 0,5 \times 0,01 \mathrm{~mm}^{3}$, forment le système de déflection du spot.

Un dispositif optique simple placé entre les deux déflecteurs assure un déroulement spatial du balayage qui a la forme d'une surface pyramidale carrée droite.

Le spot est focalisé à l'aide d'un microscope, qui permet également le contrôle visuel du positionnement sur l'échantillon. Deux polariseurs sont utilisés pour faire varier l'intensité du faisceau laser.

Les objectifs sont choisis compte tenu des dimensions de l'échantillon. Le diamètre minimum du spot est de $2 \mu \mathrm{m}$ par suite des effets de diffraction.

3. Applications. - Quelques applications montrant les possibilités du système réalisé sont brièvement présentées ici. Les images décrites ci-dessous sont obtenues pour des zones de jonctions.

Utilisant le système complet, les graphiques de la figure 3 visualisent une portion de la jonction baseémetteur d'un transistor de puissance 2 N 3055. L'ex- 


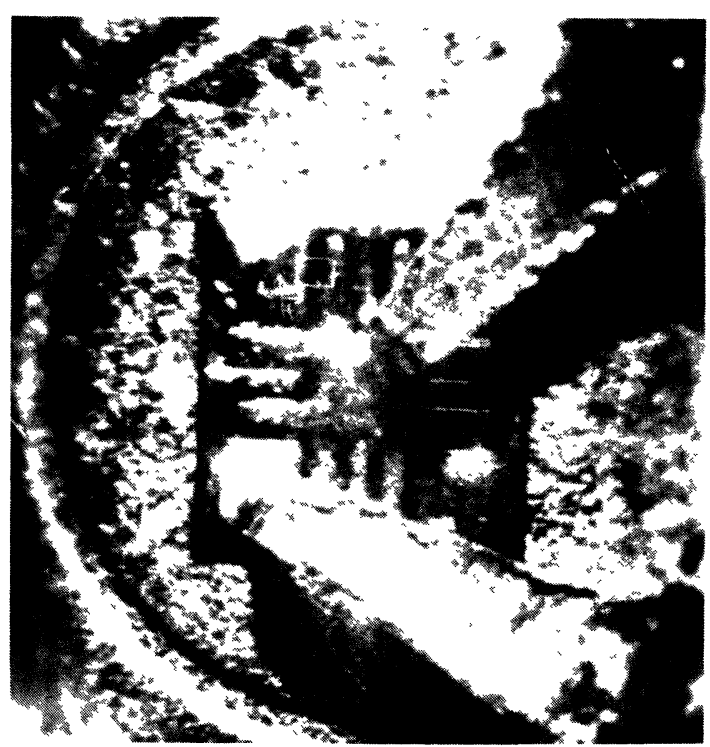

a)

Fig. 3. - Photocourant d'un transistor NPN de puissance a) Microphotographie d'une partie de la surface d'un transisto 2 N 3055. La zone explorée est marquée dans un carré. On voit auss

[Photocurrent of a power NPN transistor : $a$ ) Photomicrograph of the portion on $2 \mathrm{~N} 3055$ transistor's surface. This portion is within the indicated square. Also, it can be seen the exploration
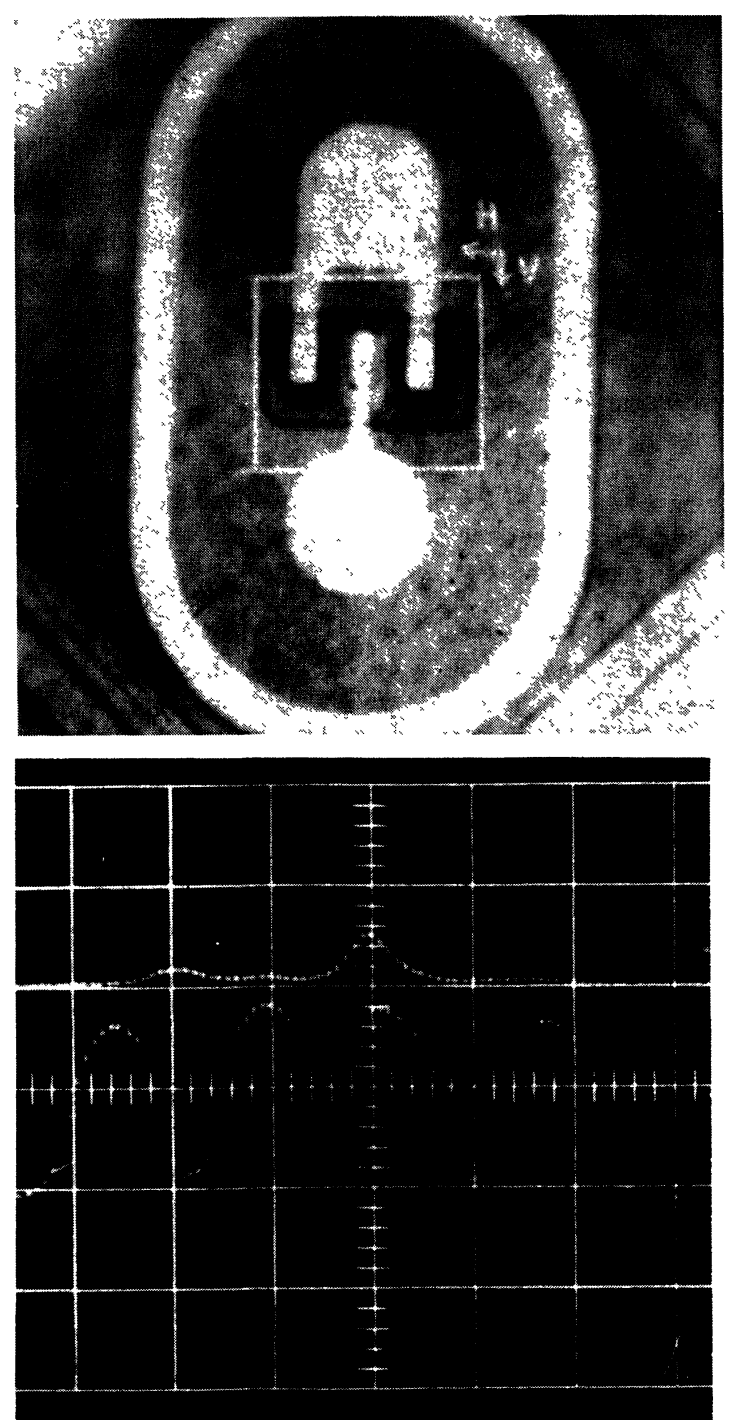

\section{b)}

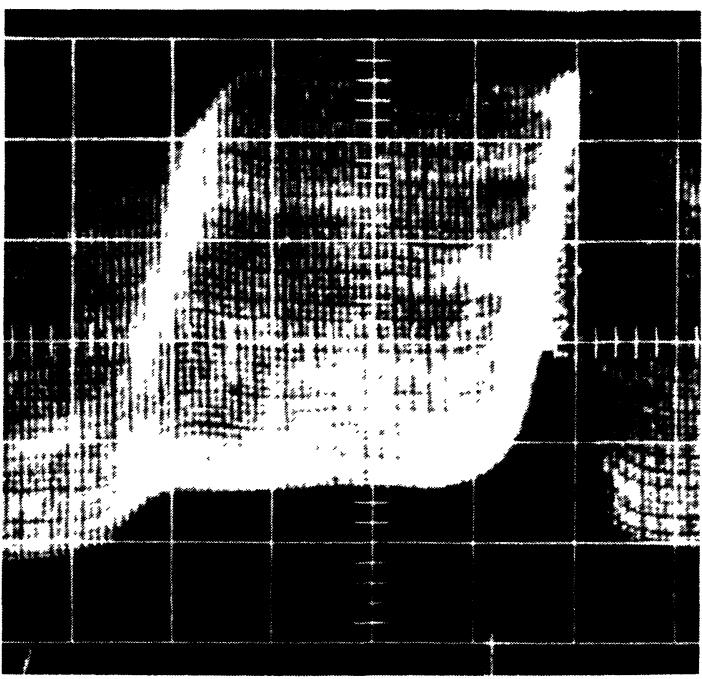

le sens d'exploration. Le balayage a été fait sur une petite région de la jonction émetteur-base. b) Photocourant du transistor non polarisé, obtenu en utilisant le système complet des graphiques.

senses. A small region of the emitter base jonction was scanned. b) Photocurrent of the transistor at $0 \mathrm{~V}$ bias, using the complete system of graphics.] a)

b)

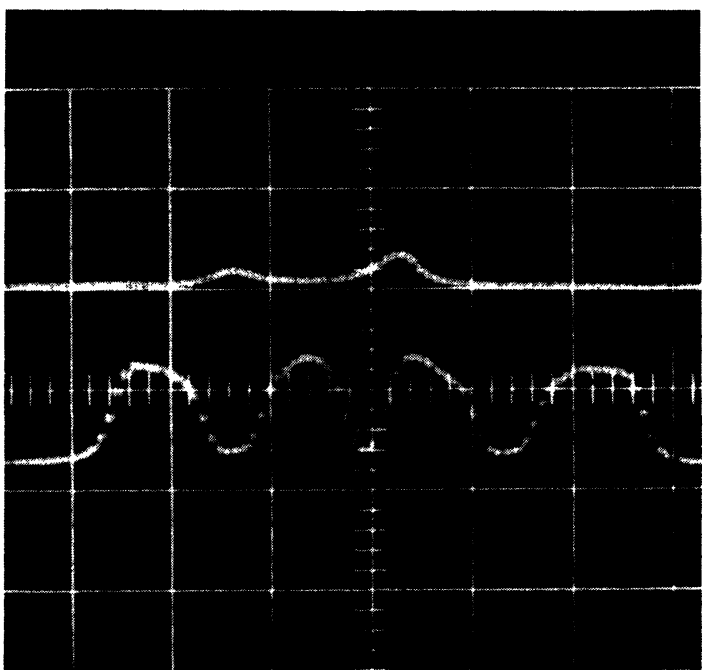

c)

d)

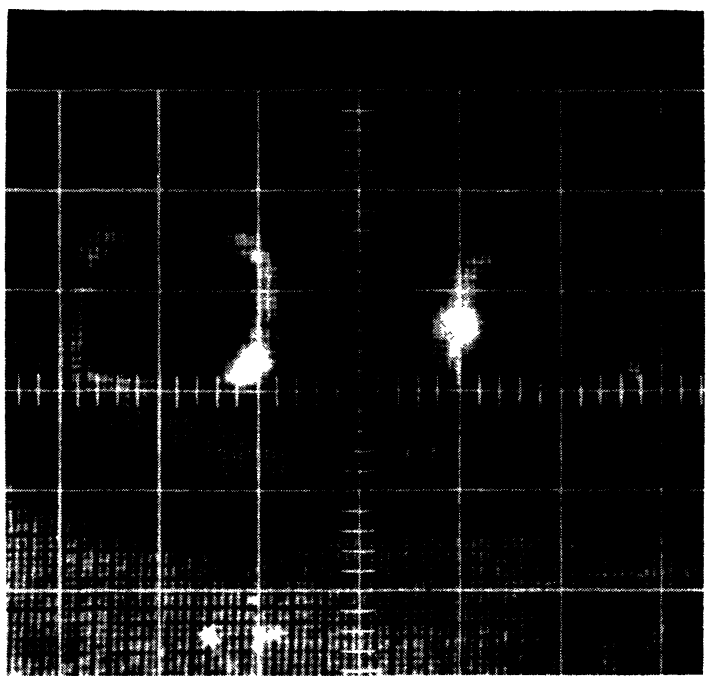

FIG. 4. 


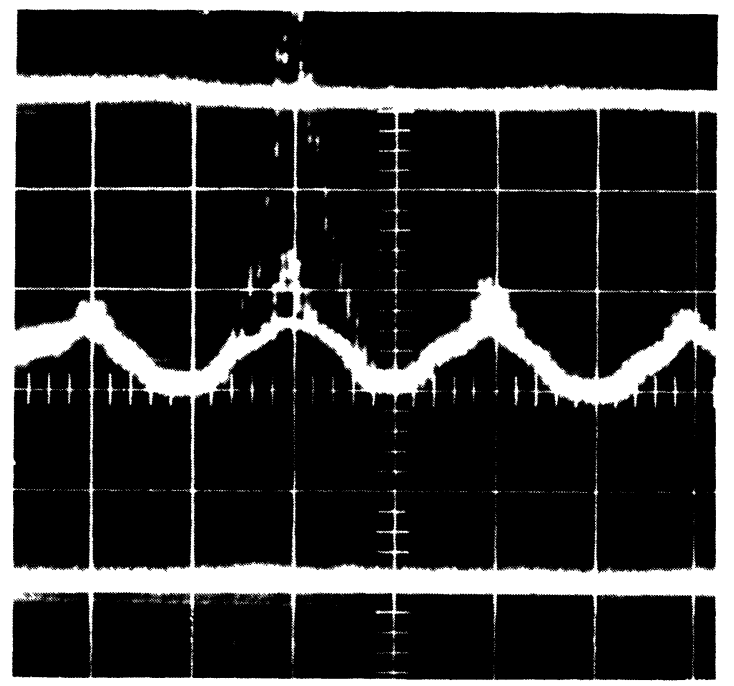

e)

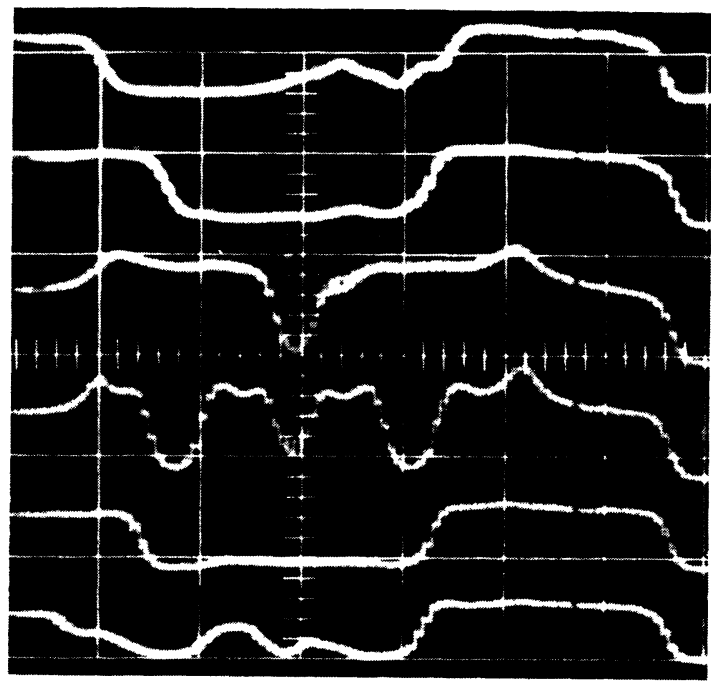

g)

pansion de la région de charge d'espace dans la base (moins dopée) est visible par le palier à gauche dans la figure $3 b$.

Un bon transistor PNP de commutation (BSX-35) est comparé à deux transistors de même type dans les figures $4 a-g$. Les figures montrent un claquage prématuré ou un canal d'inversion, qui s'observent aussi sur les diagrammes $(\mathrm{V}, \mathrm{I})$.

Nous les avons observés aussi sur les diagrammes $(\mathrm{U}, \mathrm{I})$.

A titre d'illustration sont montrées des microphotographies de dispositifs sur lesquelles sont marqués les zones explorées ainsi que les sens du balayage.

Ces figures indiquent les différents modes de fonctionnement du système : photographie (qui donne la possibilité de localisations) ou ensemble à nombre variable de graphiques (qui fournit des déterminations quantitatives).

4. Conclusions. - Dans ce travail, nous avons présenté un nouveau système de balayage à faisceau laser, applicable à l'étude des dispositifs semiconducteurs, et utilisant un laser à $\mathrm{He}-\mathrm{Ne}$ de longueur d'onde $632,8 \mathrm{~nm}$ (l'optique utilisée permettrait de travailler

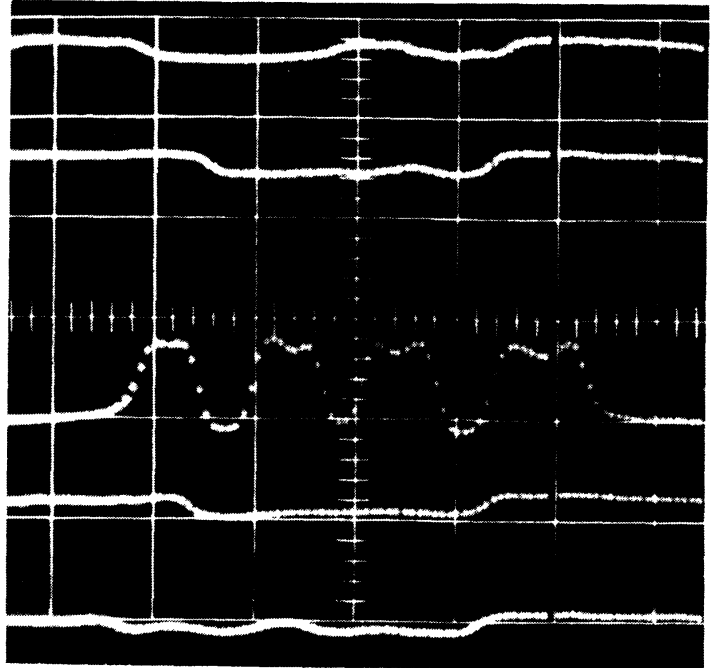

Fig. 4. - Une comparaison de réponses données par quelques transistors de commutation. Les photocourants représentés sont obtenus lorsque les dispositifs sont connectés entre la base et le collecteur. Les photographies représentent des dispositifs soit non polarisés, soit à jonction base-collecteur polarisée en inverse. a) Microphotographie de la surface du transistor BSX 35. Sur cette photographie sont indiqués la zone et les sens du balayage. b) Graphiques obtenus sur un transistor non détérioré et non polarisé. c) Même transistor qu'en $b$ ), pour une polarisation de $15 \mathrm{~V} . d)$ Microphotographie obtenue, à l'aide du système du balayage présenté, dans le cas d'un transistor à claquage prématuré. La jonction base-collecteur a été polarisée à $11,5 \mathrm{~V}$. e) Graphiques superposés, réalisés sur le même transistor qu'en $d$ ) pour quelques valeurs de la tension $(10,5 \mathrm{~V} ; 11,5 \mathrm{~V} ; 11,6 \mathrm{~V})$. On observe les différents niveaux de la multiplication correspondant aux niveaux de tension appliquée. $f$ ) Transistor à canal d'inversion non polarisé. $g$ ) Même transistor qu'en $f$ ), polarisé à $1 \mathrm{~V}$.

[Comparison among a few switching transistors. The photocurrents were obtained for the collector-base connected devices, when emitter lead was floating. The photographs shown devices having collector-base jonction at $0 \mathrm{~V}$ bias or under reverse bias. a) Micrograph of the BSX 35 surface. The region and the scanning senses are indicated. $b$ ) The graphics of a good transistor at $0 \mathrm{~V}$ bias were obtained. c) The same transistor of $(b)$ under $15 \mathrm{~V}$ reverse bias. d) A scanned micrograph of the transistor having low breakdown. Jonction there is under $11.5 \mathrm{~V}$ reverse bias. e) Graphics were obtained on the same transistor for different values of the bias $(10.5 \mathrm{~V} ; 11.5 \mathrm{~V} ; 11.6 \mathrm{~V}) . f)$ Transistor with inversion channel at $0 \mathrm{~V}$ bias. $g$ ) The same transistor of $(f)$ under $1 \mathrm{~V}$ reverse bias.]

en utilisant un faisceau de longueur d'onde différente). Les avantages du système décrit sont liés à la commande digitale et la possibilité d'utilisation d'un calculateur associé au dispositif de balayage. L'exploration de l'échantillon peut ainsi être réalisée soit manuellement soit automatiquement. Un système d'affichage assure par ailleurs le contrôle de position, en coordonnées cartésiennes, du spot sur la surface d'échantillon.

Remerciements. - Nous remercions M. D. Sachelarie pour les dispositifs semiconducteurs et les discussions. 


\section{Bibliographie}

[1] Tihanyi, J., Pasztor, G., Solid State Electron 10 (1967) 235. [6] McMahon, R., Electronics 4 (1971) 92.

[2] Haberer, J., Phys. Failure Electron. 5 (1967).

[3] Lile, D. L., Davis, N. M., S.S.E. 18 (1975) 699.

[4] Potter, C. N., Sawyer, D. E., Rev. Sci. Instrum. 392 (1968)

[7] Sherman, B., Black, J. F., Appl. Opt. 9 (1970) 802. 180.

[8] Black, J. F., Summers, C. J., Sherman, B., Appl. Opt. 11 (1972) 1553.

[5] Phelan, R. J. Jr., De Meo, N. L. Jr., Appl. Opt. 104 (1971)

[9] Popescu, I. M., Stanciu, G. A., Stoichita, C. M., Bul. Inst. 858 . 